Apidologie, 1977, 8 (2), 101-109.

\title{
SUR L'ORIGINE DES RÉSINES EMPLOYÉES PAR HERIADES TRUNCORUM L. (HYMENOPTERA, MEGACHILIDAE) POUR LA CONSTRUCTION DE SES NIDS
}

\author{
$\ddot{U}$ ber die Herkunft der von Heriades truncorum L. (Hymenoptera, \\ Megachilidae) beim Bau ihrer Nester verwandten Harze
}

\author{
Maria de Lourdes MAGIEL DE ALMEIDA CORREIA* \\ Laboratoire d'Éthologie et d'Écologie des Insectes, I.N.R.A., \\ 86600 Lusignan
}

\section{SUMMARY}

ON THE ORIGIN OF RESINS USED BY Heriades truncorum L.

(Hymenoptera, Megachilidae) FOR NEST BUILDING

For nest building females of the hymenoptera Heriades truncorum L. use a resin of which origin has not been specified up to now. In this work it is shown that the stuff used contains a range of components which have the same chromatographic behaviour as those found in a conifer resin Pinus Laricio L., a frequent species in the area.

Analyses were made on 88 nests collected through 4 years and in 8 different geographic positions. Extracts of plugs and cell walls were chromatographied on fluorescent silicic acid thin-layer in three development systems. Chromatograms were observed in ultraviolet light.

So females of Heriades truncorum L. are likely to gather conifers resin to build their nests. This has not been yet directly observed. The present status of researches does not allow to exclude the possibility of some substances produced by the insect being then added to products of plant origin.

* boursière de la Fondation Calouste Gulbenkian. 


\section{RÉSUMÉ}

Les femelles de l'Hyménoptère Heriades truncorum L. utilisent pour la construction de leur nid, une résine dont l'origine n'avait pas été précisée jusqu'à ce jour. Dans le présent travail, nous montrons que le matériel employé contient une série de constituants de même comportement chromatographique que ceux qui sont présents dans la résine du Conifère Pinus Laricio L., espèce fréquente dans la région.

Les analyses ont porté sur 88 nids collectés au cours de 4 années et dans 8 situations géographiques distinctes. Les extraits de bouchons et de cloisons ont été chromatographiés sur couche mince d'acide silicique fluorescent dans trois systèmes de développement. Les chromatogrammes sont observés en lumière ultraviolette.

Il est donc vraisemblable que les femelles de Heriades truncorum $\mathrm{L}$. récoltent la résine de Conifères pour la construction de leurs nids. L'observation directe du phénomène n'a pas encore été faite. L'état actuel des recherches ne permet pas d'éliminer la possibilité que certaines substances produites par l'insecte soient ensuite ajoutées aux produits d'origine végétale.

\section{INTROD UCTION}

La récolte de produits utilisés par les femelles de l'espèce Heriades truncorum L. pour cloisonner et boucher les nids est une activité saisonnière. Éléments essentiels dans la construction de ces derniers, les bouchons et les cloisons sont formés de mélanges assez complexes, possédant une consistance et une coloration variable (jaune très clair, jaune foncé, orange ou noir) mais ayant la texture et l'odeur de résine de Conifère.

Le procédé de construction est le même pour toutes les cellules : la femelle construit d'abord le bouchon initial avec un produit résineux ou cireux et tapisse légèrement la séparation des deux parties horizontales du tube. Ensuite elle approvisionne la cellule, c'est-à-dire accumule du pollen, dépose l'œuf et la cloisonne avec une matière apparemment identique à celle des bouchons. A la fin elle ferme le tube avec un bouchon terminal dont la nature est parfois différente de celle des bouchons initiaux.

Nous avons donc cherché à préciser la provenance de ces matériaux de construction et à déterminer leur nature par chromatographie sur couche mince.

\section{MÉTHODES ET TECHNIQUES}

\section{Mode opératoire}

De chaque nid, à l'aide d'une spatule très fine sont retirés séparément quelques milligrammes du bouchon initial, du bouchon terminal et des cloisons en évitant leur contamination par les provisions ou les autres produits présents. 
Après avoir testé la concentration convenable par un essai préalable, on dilue chaque échantillon dans $0,30 \mathrm{ml}$ de chloroforme. La solution est homogénéisée pendant cinq à dix minutes. Des extraits de résines végétales (bois broyé et gommes), ainsi que du mastic à greffer, trouvés dans les différents endroits de piégeage sont dissous dans le même solvant, constituant ainsi nos témoins.

L'étude des composés chimiques constituant ces produits, oxydés à l'air et qui se comportent comme des mélanges complexes, demande beaucoup de temps et n'est pas simple. Aussi avons-nous limité notre étude à l'observation. chromatographique sur couches minces effectuées sur films d'acide silicique fluorescents Schleider-Schull.

Les substances à chromatographier sont alors déposées sous forme de taches, sur une ligne horizontale à $2 \mathrm{~cm}$ de l'extrémité inféricure de la feuille de gel de silice. Chaque tache du mélange à analyser est déposée au moyen d'une micro-pipette Pasteur à $1,5 \mathrm{~cm}$ de la précédente. Sur une même feuille de gel de silice et sur une même ligne horizontale, nous avons simultanément effectué le dépôt des échantillons du mélange à analyser et des échantillons de solutions de substances témoins.

Après séchage des taches ainsi obtenues, l'extrémité de la feuille est plongée dans une cuve chromatographique dont l'atmosphère a été saturée avec le solvant $(100 \mathrm{ml})$. Opérant dans une série de systèmes différents, le développement est effectué par l'éther de pétrole $\left(30-60^{\circ}\right)$, par l'éther de pétrole-acétate d'éthyle dans la proportion $7: 3$, et l'éther de pétrole-acétate d'éthyle dans la proportion $1: 1$. Trois cuves contenant les éluants précédents ont été utilisées. Pour chaque solution du mélange inconnu et du témoin recueilli dans la nature, nous avons ainsi réalisé trois séries de chromatogrammes (fig. 1).

L'élution est arrêtée lorsque le déplacement du front du solvant arrive à $4 \mathrm{~cm}$ du bord supérieur de la plaque. Celle-ci est alors séchée à l'air pendant $5 \mathrm{mn}$. Les substances échelonnées à des niveaux différents sont ensuite mises en évidence au moyen d'un appareil U.V. (observation à $254 \mathrm{~nm}$ et $366 \mathrm{~nm}$ ) placé dans une petite chambre noire.

Nous obtenons ainsi un ensemble de taches, comparable à une empreinte, caractéristique du mélange étudié et qu'il est possible de comparer à des extraits de résine de conifères. La retranscription directe des différentes taches est effectuée sur papier calque pour observation et comparaison ultérieure des chromatogrammes.

\section{RÉSULTATS, DISCUSSION ET CONCLUSIONS}

Si incomplètes que soient nos données sur l'analyse chromatographique, elles montrent qu'il est possible d'effectuer un classement en catégories sur la base du schéma typique de la résine de pin Pinus Laricio L. 


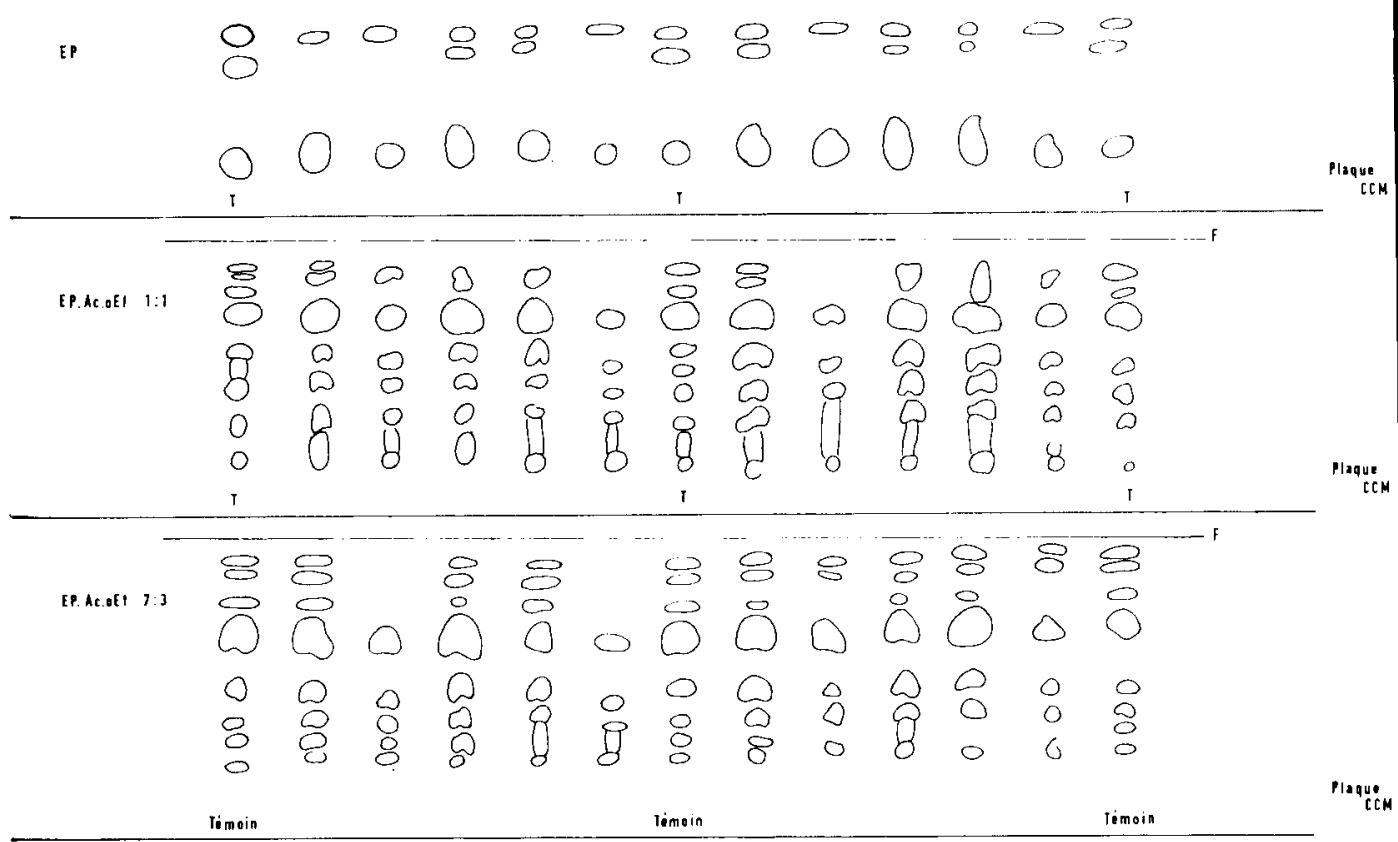

FIG. 1. - Chromatographies sur couches minces.

F : front du solvant

EP : éther de pétrole

EP.Ac.oEt : Ether de pétrole-acétate d'éthyle dans la proportion de $1: 1$ et $7: 3$

$\mathrm{T}$ : témoin. Les autres taches correspondent aux mélanges à analyser.

Aв8. 1. - Dünnschicht-Chromatographien.

F $\quad=$ Pik des Lösungsmittels

EP $=$ Petroläther

EP.Ac.oEt = Petroläther-Äthylacetat im Verhältnis von $1: 1$ und $7: 3$

$\mathbf{T}=$ Kontrolle. Die anderen Flecken entsprechen den zu analysierenden Mischungen

De façon à permettre le regroupement des chromatogrammes, un code à été établi de la façon suivante :

o - rien de visible en U.V. sous les deux longueurs d'o nde.

$\mathrm{F}$ - forte fluorescence à $366 \mathrm{~nm}$.

A - schéma typique de la résine de pin : Pinus Laricio L.

$\mathrm{A}^{\prime}$ - schéma voisin de celui qui est fourni par la résine de pin : Pinus Laricio L.

B - schéma ne correspondant à aucun des matériaux essayés comme témoins.

FA - schéma typique et forte fluorescence à $366 \mathrm{~nm}$.

$\mathrm{FA}^{\prime}$ - schéma voisin du type $\mathrm{A}^{\prime}$ et forte fluorescence à $366 \mathrm{~nm}$. 
Récoltées dans les endroits de piégeage, différentes substances ont été essayées (tableau I.)

TABL. 1. - Comportement en lumière ultra-violette des extraits chloroformiques de divers matériaux ramassés dans les lieux de piégeage.

TAB. 1. -- Verhalten der Chloroformextrakte verschiedener an den Nestnahmeplätzen gesammelter Stoffe.

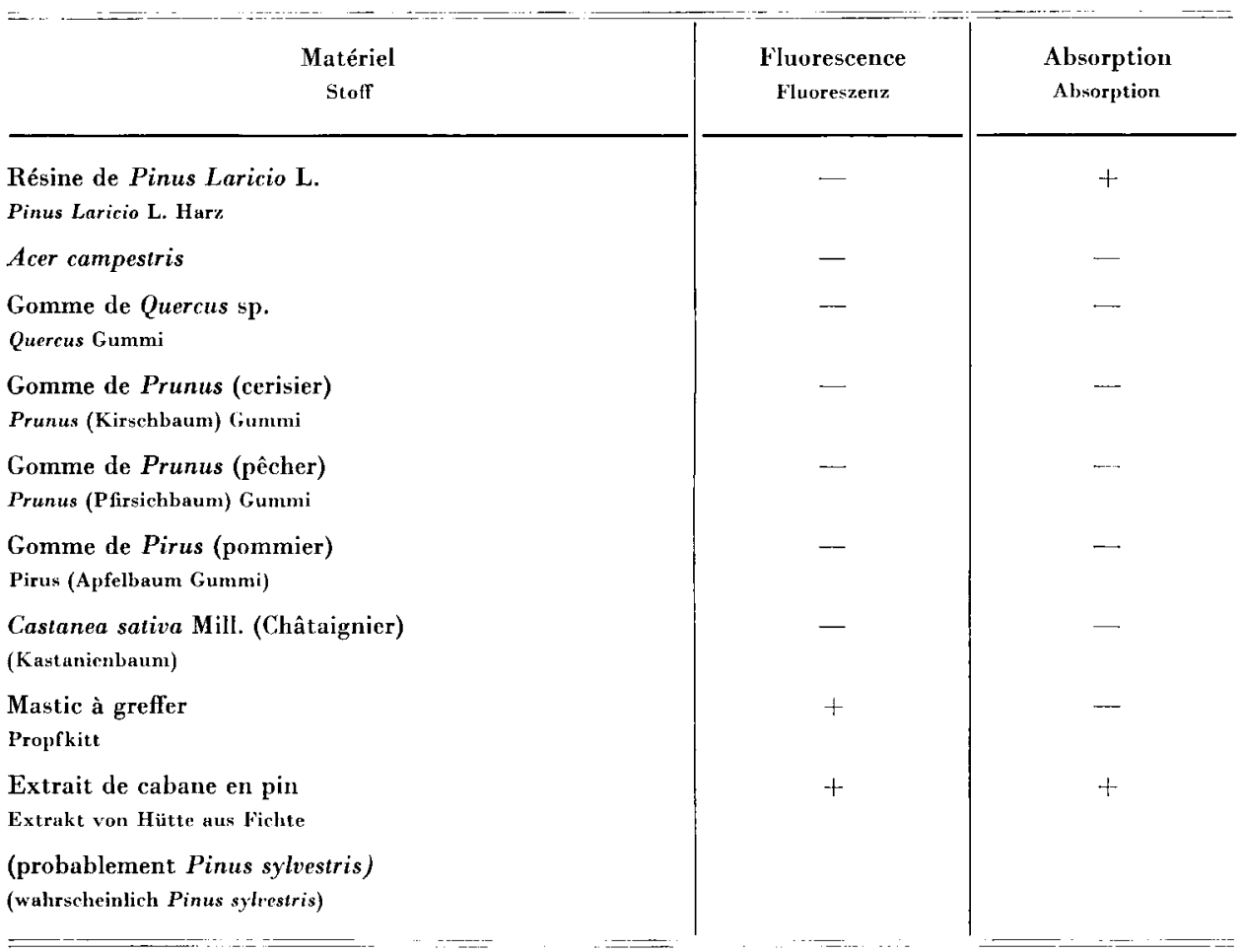

Il faut noter que les extraits de cabane en planches de pin peintes et de mastic à greffer ont donné des spots fluorescents à $366 \mathrm{~nm}$. Nous considérerons d'abord la répartition des schémas chromatographiques globalement, nous essayerons ensuite d'observer les problèmes particuliers, tels que la répartition suivant les années.

Le tableau 2 montre comment les différents types de chromatogrammes se répartissent dans les endroits de piégeage : Barbezieux, Le Chêne, GrandBreuil, Jazeneuil, Mongadon, La Pétinière, Servolet et les Verrines.

Au Grand-Breuil et Servolet seul le schéma A est présent. Au Chêne et aux Verrines un deuxième, le $A^{\prime}$, est aussi présent. Les autres localités : Mongadon, Jazeneuil (La Girauderie) et la Pétinière présentent une gamme plus diversifiée 
de chromatogrammes. Toutefois, on constate une forte prédominance du schéma typique de résine de Pinus Laricio L. dans les zones de Mongadon et de Jazeneuil (La Girauderie).

On remarque également que la distribution régionale de la Pétinière donne des résultats proches de ceux de Mongadon pour les schémas dominants. Dans les deux endroits, les chromatogrammes type A, A', FA, sont présents; pour Mongadon trois schémas supplémentaires apparaissent $\mathrm{O}, \mathrm{F}, \mathrm{FA}^{\prime}$, alors que pour la Pétinière seulement le schéma $B$ apparaît. Sur les chromatogrammes de Mongadon des taches fluorescentes sont visibles en longueur d'onde de $366 \mathrm{~nm}$, alors que toutes les autres le sont en $254 \mathrm{~nm}$. La Pétinière est le seul endroit où nous remarquons un schéma ne correspondant à aucune des résines utilisées comme témoins. Les deux lieux La Girauderie et Mongadon se singularisent par une fréquence relativement forte du schéma typique $A$ et par le peu de schémas de type 0 . Ce sont d'ailleurs les seuls endroits où ce dernier est remarqué. Au Chêne et aux Verrines sont présents des schémas voisins des types $A$ et $A^{\prime}$. Le schéma $A^{\prime}$ si voisin de celui fourni par la résine de Pin, serait-il dû à un apport de substance sécrétée par les femelles ou s'agit-il d'une autre espèce de Conifère?

Après cet aperçu global de la répartition des schémas chromatographiques des divers endroits de piégeage étahlie pour l'ensemble des années 1972, $1973,1974,1975$, il serait intéressant de voir si la nature des matériaux utilisés par les Heriades a varié au cours de ces quatre années (Voir tableau 2, et fig. 2). Le schéma chromatographique $A$ domine très nettement : il est présent chaque année et a été observé dans toutes les localités explorées et sa fréquence est plus élevée que celle de tous les autres schémas. Nous remarquons également que le schéma $A^{\prime}$ voisin du précédent est apparu dans six endroits; tous les autres schémas n'ont été observés au cours des 4 années que dans une ou deux localités au maximum. Pour les années 1972 et 1974 la présence des taches fluorescentes est nette, avec un assez grand pourcentage pour 1974. Il serait intéressant d'analyser cette fluorescence.

Le schéma chromatographique A qui est le plus fréquent est tout à fait semblable au schéma fourni par la résine de Pinus Laricio L. Ceci nous amène à penser que les Heriades utilisent la résine de ce conifère pour édifier leur nid. Cependant il n'est pas exclu que la résine d'autres genres ou espèces de Conifères présentent un schéma chromatographique comparable. Nous aurons done à compléter cette étude par une analyse chromatographique en phase gazeuse à la fois des constructions d'Heriades et des résines des divers conifères présents dans la région. Une fois connue l'exigence floristique d'Heriades truncorum L. nous espérons maîtriser la nidification de cet insecte dans des conditions semi-artificielles. 


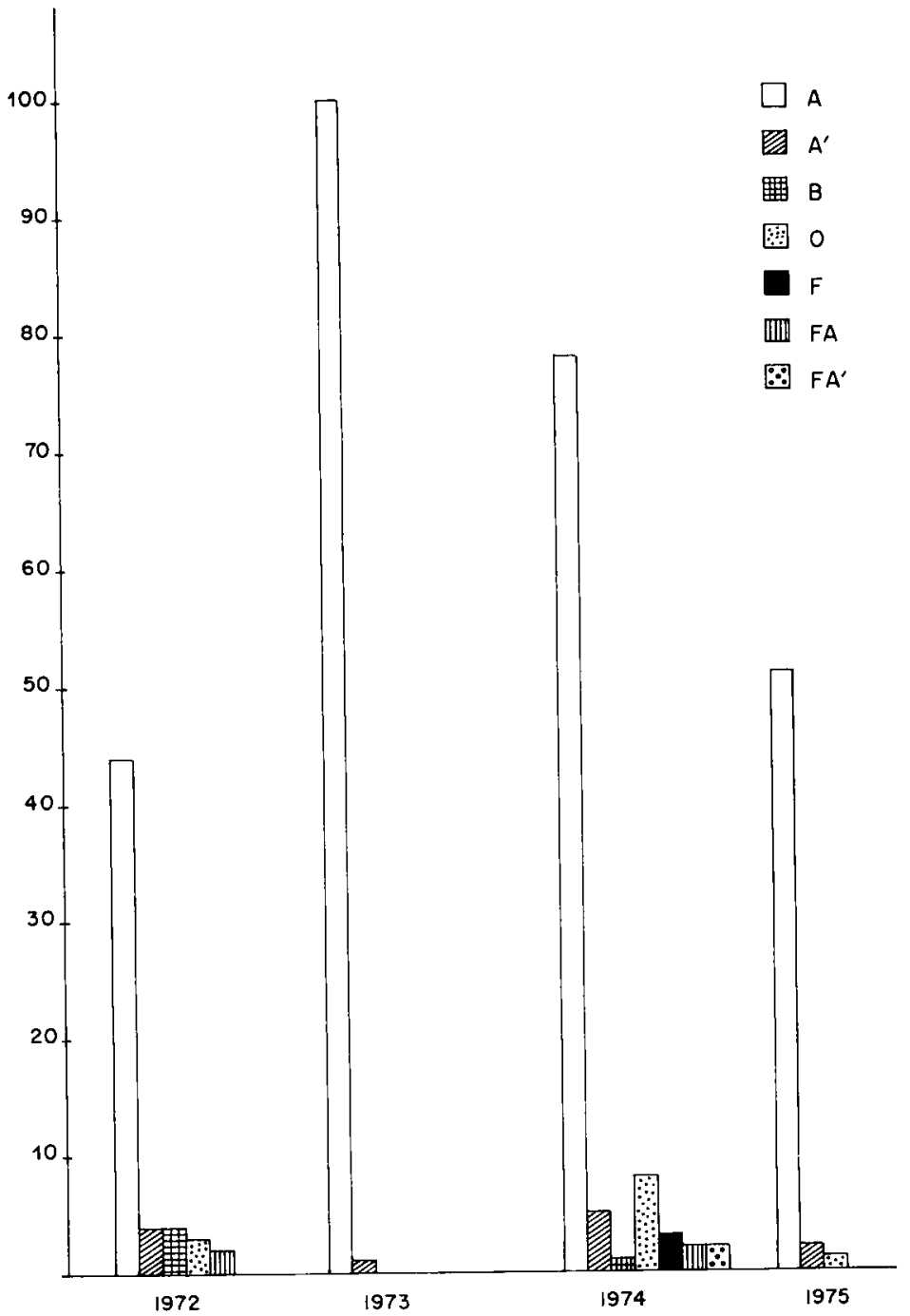

FIG. 2. - Répartition des schémas chromatographiques selon les quatro années.

Авв. 2. - Verteilung der chromatographischen Schemata in bezug auf die vicr Jahre.

A - schéma typique de la résine de pin : Pinus Laricio L.

A = Typisches Schema von Pinusharz (Pinus Laricio L.).

$A^{\prime}$ - schéma voisin de celui fourni par la résine de pin : Pinus Laricio L.

$\mathbf{A}^{\prime}=$ Schema, dem von Pinusharz benachbart.

B - Schéma ne correspondant à aucun des matériaux essayés comme témoins.

$\mathrm{B}=$ ein Schema, das keinem der Kontrollstoffe entspricht.

$\therefore \because \quad 0 \quad-$ rien de visible en U.V. sous les deux longueurs d'onde.

$\therefore: \therefore \quad 0=$ unter beiden Wellenlängen nichts im $\mathrm{UV}$ zu sehen.

F - forte fluorescence à $366 \mathrm{~nm}$.

$\mathrm{F}=$ starke Fluoreszenz bei $366 \mathrm{~nm}$.

FA - schéma typique et forte fluorescence à $366 \mathrm{~nm}$.

FA = Typisches Schema, bei $366 \mathrm{~nm}$ stark fluoreszierend.

$F^{\prime} A^{\prime}$ - schéma voisin du typique et forte fluorescence à $366 \mathrm{~nm}$.

$\mathbf{F A}^{\prime}=$ ein dem vorigen benachbartes Schema. 


\section{REMERCIEMENTS}

Je remercie bien vivement M. Barbier - Institut de Chimie des substances naturelles, C.N.R.S., Gif-sur-Yvette - pour l'accueil qu'il m'a réservé dans son laboratoire et son aide dans la réalisation de ce travail.

J'adresse aussi mes remerciements à MM. S. Carré et P. Cantot pour l'aide apportée dans la récolte des substances témoins.

\section{ZUSAMMENFASSUNG}

Wichtige Bestandteile der Nestkonstruktionen von Heriades truncorum L., die Verschlusspfropfen und die Scheidewände, bestehen aus ziemlich komplexen Mischungen und sind von verschiedener Konsistenz und Färbung, besitzen jedoch das innere Gefüge und den Geruch von Koniferenharz.

Verf. hat versucht, die Herkunft dieser Baustoffe genau festzulegen und ihre Natur durch Dünnschicht-Chromatographie auf fluoreszierendem Kiesel-Gel nach Schleicher-Schüll zu bestimmen. Die Analysen erstreckten sich auf $88 \mathrm{im}$ Verlauf von vier Jahren an acht geographisch verschiedenen Orten gesammelte Nester.

Um Vergleiche zu ermöglichen, wurden pflanzliche Harze (zerriebenes Holz und Gummi) sowie Kittharz, die alle an den verschiedenen Plätzen der Nestnahme gesammelt wurden, ebenfalls chromatographisch untersucht. Es zeigte sich, dass das von Heriades verwandte Material eine Reihe von Bestandteilen besitzt, die das gleiche chromatographische Verhalten aufweisen, wie das Harz der Konifere Pinus Laricio L., einer in der Gegend häufigen Art.

Das chromatographische Schema $A$, das am häufigsten vorkommt (Tab. 2 Abb. 2), stimmt mit dem des Lärchenharzes (Pinus Laricio L.) vollkommen überein. Das lässt darauf schliessen, dass Heriades beim Nestbau das Harz dieser Konifere benutzt. Es ist indessen nicht ausgeschlossen, dass das Harz anderer Gattungen oder Arten von Koniferen ein ähnliches chromatographisches Schema aufweist. Wir müssen daher diese Untersuchung durch gleichzeitige gas-chromatographische Untersuchungen des Nestbau-Materials von Heriades und der Harze der in der Gegend vorkommenden Koniferen vervollständigen. Wenn die Erfordernisse von Heriades truncorum in bezug a uf die Pflanzen bekannt sind, können wir hoffen, den Nestbau dieses Insekts unter halbkünstlichen Bedingungen zu bewerkstelligen.

\section{RÉFÉRENCES BIBLIOGRAPHIQUES}

Maciel de Almeida Correia (Maria de Lourdes), 1976. - Fluctuations de populations naturelles chez les genres Chelostoma et Heriades (Hymenoptera, Megachilidae). Apidologie, 7 (2), 189-195.

Magiel de Almeida Correia (Maria de Lourdes), 1976. - Notes sur la biologie d'Heriades truncorum Linn. (Hymenoptera, Megachilidae). Apidologie, 7 (2), 169-187.

Savidan L., 1970. — La chromatographie - Dunod, Paris. 